\title{
COVID-19: time to flatten the infodemic curve
}

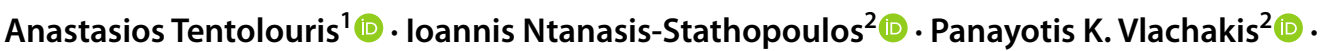 \\ Diamantis I. Tsilimigras ${ }^{3}$ (]) Maria Gavriatopoulou ${ }^{2}$ (1) - Meletios A. Dimopoulos ${ }^{2}$ (1)
}

Received: 20 November 2020 / Accepted: 15 December 2020 / Published online: 8 January 2021

(c) The Author(s), under exclusive licence to Springer Nature Switzerland AG part of Springer Nature 2021

\begin{abstract}
Thousands of articles have been published regarding the coronavirus disease of 2019 (COVID-19). Most of them are not original research articles but reviews and editorials, and therefore, the absence of evidence-based guidelines has been evident. In parallel, the quality of manuscripts is questionable since the number of preprints has increased due to the need of fast publication of COVID-19-related articles. Furthermore, the number of retracted articles during the pandemic is exceptionally high. Media have an important role in the distribution of incorrect information, nevertheless individual people and policy makers are also responsible. As misinformation thrives in crisis periods, well-designed studies are needed to flatten the infodemic curve regarding prevention, diagnosis, and long-term complications of COVID-19.
\end{abstract}

Keywords COVID-19 $\cdot$ SARS-CoV-2 $\cdot$ Misinformation $\cdot$ Infodemic $\cdot$ Social media

\section{Introduction}

The novel coronavirus disease of 2019 (COVID-19) is a global pandemic with $54,301,156$ cases and 1,316,994 deaths worldwide up to 16 November 2020 [1]. Misinformation, which is defined as false information, shared without knowledge that it is false, and disinformation, which is fabricated information distributed with the clear intention to mislead, is commonly observed in this era [2]. In the beginning of the COVID-19 pandemic, Dr. Tedros Adhanom Ghebreyesus, the Director-General of the World Health Organization (WHO), stated that "we're not just fighting an epidemic; we're fighting an infodemic" [3]. The term infodemic has been used to outline the hazards of misinformation during

Maria Gavriatopoulou

mariagabria@gmail.com

1 First Department of Propaedeutic Internal Medicine, School of Medicine, National and Kapodistrian University of Athens, Laiko General Hospital, Athens, Greece

2 Department of Clinical Therapeutics, School of Medicine, National and Kapodistrian University of Athens, Alexandra General Hospital, 80 Vas. Sofias Avenue, 11528 Athens, Greece

3 Division of Surgical Oncology, Department of Surgery, Wexner Medical Center, The Ohio State University, James Comprehensive Cancer Center, Columbus, OH, USA the management of disease outbreaks, since it could negatively affect the social response to the pandemic.

This is not the first time that false information is spreading in a health crisis. During the Ebola outbreak in the Democratic Republic of Congo in 2019, misinformation led to violence, social disturbances as well as targeted attacks on health care providers [4]. Throughout the 2003 severe acute respiratory syndrome (SARS) epidemic in the USA, fear, stigmatization, and discrimination were particularly evident among Asian-American communities [5]. In addition, 40 years after the beginning of the human immunodeficiency virus (HIV) global epidemic, fear related to prejudice and racism still negatively affects public health efforts [6].

\section{Quantity of data for COVID-19}

COVID-19 has provided fertile ground for publication of scientific papers. A report that examined the number of published articles in the first 3 months of the pandemic, starting from January 2020, showed that within a period of 92 days a total of 3201 articles were published on this topic, and within 113 days, 6831 articles were published; with an average of 34.8 articles and 58.89 articles per day, respectively, this may be the highest number for any disease so far [7]. We performed a literature search in the PubMed database from the beginning of the pandemic until 15 November 
2020 to assess the number of published articles. The search term was "COVID or COVID-19 or SARS-CoV-2". Overall, more than 74,300 articles have been published within 11.5 months. When comparing the number of publications in PubMed between COVID-19 and influenza, the numbers are impressive, since a total of 17,559 articles on COVID19 were published in the period between February and June 2020, while only 2316 papers were retrieved with the term "H1N1" in the period of June 2009 to May 2010 - that is an entire year of the Influenza A H1N1 [8]. Since the previous study for influenza was made in a different period, we additionally performed a search in the PubMed using the term "influenza" between 1 January 2020 and 15 November 2020 and a total of 6757 articles were found. We also assessed the type of articles on COVID-19 using the filters of the PubMed database. The number of published original research papers $(n=4841)$ was by far lower than the number of reviews, editorials, or meta-analyses $(n=13,890)$. Another study has examined the research methodology of journal articles that were published from 1 December 2019 until April 2020 on peer review journals and showed that among the first 2118 articles on COVID-19, only 533 (25\%) of them contained original data [9]. In terms of type of research articles, among 312 articles that self-reported the study design, the most common were retrospective studies $(n=88,28 \%)$ and case reports $(n=86,28 \%)$ [9]. Other type of studies included case series $(n=46,15 \%)$, modelling studies $(n=18,5.7 \%)$, systematic reviews with or without meta-analysis $(n=16,5.1 \%)$ and $58(18.6 \%)$ consisted of other type of articles.

Randomized controlled trials (RCTs) are considered the "gold-standard" to evaluate the efficacy and safety of novel treatments. However, in the beginning of the pandemic most of the articles on COVID-19 were observational which is reasonable, since interventional studies usually require more time to be completed [9]. Even though evidence-based medicine is not limited to RCTs and meta-analyses, the lack of original research articles and especially the lack of RCTs has led to the absence of evidence-based guidelines. It should be noted that the COVID-19 pandemic is still ongoing and well-designed studies are difficult to be performed in practice, since the number of infected people has been increasing dramatically and hospitals are overcrowded with patients. Therefore, there is lack of both time and personnel to perform well-designed interventional studies.

\section{Quality of data for COVID-19}

\section{Preprints}

Another issue apart from the lack of well-designed studies on COVID-19 is the quality of data. During the outbreak of
Fast distribution of new information worldwide Preventive measures

Communication of healthcare leaders

Collaborative research projects

Continuing medical education

Optimization of therapeutic strategies

Advantages

COVID-19 PANDEMIC

Disadvantages

Lack of RCTs
Lack of evidence-based guidelines
Poor peer-review quality
High number of retractions
(Social) Media misinformation

Fig. 1 Two conflicting sides of global distribution of information about COVID-19 pandemic. COVID-19: Coronavirus disease of 2019; RCTs: Randomized controlled trials

COVID-19, peer review has been virtually eliminated since many articles were published in preprints. Even though peer review is fundamental for the validation of the data, the ongoing pandemic has increased the speed of publication with preprints. Preprints are publicly accessible scientific manuscripts that have not yet been certified by peer review and their basic purpose is to make new scientific information freely available before the peer review process [10]. Speed is crucial in a pandemic, especially when considering that the time for review has increased the past years [11]. For example, the median time for review at Nature publishing group journals has increased from 85 days to more than 150 days during the past decade [11]. Nevertheless, publication of preprints is based exclusively on the requirement that they are scientific and there is no guarantee that the study is well designed or that the conclusions reached are supported by the data presented [10]. Moreover, no method exists to screen preprint submissions for conflicts of interest [10]. The basic philosophy of preprints is that errors will get fixed as the scientific community comments on the findings and that the advantages of rapid sharing among scientists balance the disadvantages of sharing invalid findings.

In the beginning of the pandemic, an analysis on search trends and news media data showed that preprints-rather than peer reviewed articles-may be driving discourse related to the ongoing COVID-19 outbreak [12]. However, 
preprints were also affecting optimal treatment management, since on January 2020, WHO announced that a repository of relevant studies would be created-including those that have not yet been peer-reviewed [13]. The number of preprints has increased over $400 \%$ during the COVID-19 pandemic (from 586 for the last 15 weeks of 2019 to 2572 for the first 15 weeks of 2020) [14]. Although scientists are aware that preprint manuscripts should be taken with caution, the public or the media cannot understand the difference. Moreover, many preprints are cited by reviews and as a result, invalid information may be perpetuated.

On the other hand, articles that have been assessed via the peer review process are not necessarily of better quality than preprints. Many journals provide fast-tracked services for articles on COVID-19 and consequently the robustness of critical examination of submissions may be compromised [15]. A study that analysed 8455 articles on COVID-19 indexed in the PubMed database found that $8 \%$ of the articles had been reviewed and accepted for publication on the day they were submitted or the day after, suggesting that in some cases, the peer review process had been rushed [16].

With the fast-tracking science that is promoted with preprints and with fast-track peer review process, there is potential for wider dissemination of poor-quality work. This particular disadvantage has been emphasized during the outbreak, especially after the withdrawal of a virology study from a preprint server, which claimed that COVID19 contained HIV “insertions" [17]. In addition, when the subject is of great public interest preliminary, minimally reviewed information is spreading as fast as the virus itself. A typical example is the hydroxychloroquine hypothesis [18]. Hydroxychloroquine was the cornerstone therapy for patients with COVID-19 in the beginning of the pandemic [18]. Its use was recommended by several guidelines based on in vitro studies and non-randomized trials, while later studies demonstrated no efficacy [19].

\section{Retractions}

Similarly, another result of poor fast-track peer review process is the retractions. According to the Retraction Watch online page, a total of 38 COVID-19 manuscripts have been retracted, and some among them were published in well-known medical journals-including The Lancet and The New England Journal of Medicine [20]. The response from the Lancet journal was instant as the editors announced changes in the peer review requirements [21]. An editorial that was published in August showed that among 26 retracted articles on COVID-19, most of them $(8,30.8 \%)$ were retracted due to concerns, issues, or errors in the results and/or conclusions, reflecting the rush to quickly publish these articles [22]. Some of the retracted papers were flawed, since inappropriate statistical techniques were used or conclusions were not adequately supported by the data, while others were fraudulent.

A study that was published in June 2020 compared the retraction rate between papers for COVID-19 and for other viruses that caused a world health emergency [8]. The retraction rate was $0.097,0.024,0.023,0.023$, and 0.024 for COVID-19, Ebola, H1N1, HIV, and the Middle East respiratory syndrome (MERS), respectively [8]. However, it should be noted that direct comparisons between different viruses and different periods cannot be performed. HIV is a pandemic with 40 years duration, while regarding Ebola virus there were many outbreaks since it was first identified in 1976; however, the major occurred from December 2013 to January 2016. The main reason for the high retraction rate is the rush for fast publication of papers. The retraction rate is estimated to be even higher in the future, since retraction of articles and particularly fraudulent articles takes time (median time from publication to retraction: 28 months) [23]. Another reason for the high retraction rate is that funding availability and career opportunity tend some nonspecialist researchers to switch fields to work on the "hot topic" [8]. Last but not least, the quality of peer reviewers is questionable, especially when many experienced researchers were concentrated on their research and they did not have enough time to peer review.

\section{The role of (social) media}

During the pandemic, traditional media such as the television, newspapers, and social media have been used as a source of information for the public. The use of media during the pandemic has several advantages such as fast distribution of new information and communication of health care leaders with the public, but also has several disadvantages (Fig. 1). The enormous amount of information that is being shared cannot be easily filtered and fake news as well as inaccurate information may spread faster and wider than fact-based news [24]. Moreover, during COVID-19 there is an increasing spread of information across social media platforms such as Facebook and Twitter even by the official health care agencies after social distancing measures were put into place; therefore, the impact of poor conducted studies, preprints, and retracted papers on the scientific and non-scientific community is not negligible. Typical examples of misinformation include several cases of overdose of chloroquine in Nigeria after news reported effectiveness of the drug [25], as well as panic for buying fuel, groceries, and paper products in the USA after rumours about a national lockdown [26]. In addition, false information during the COVID-19 pandemic caused drug shortage. A real-world study in Greece that examined to which extent patients with autoimmune inflammatory 
rheumatic diseases altered their treatment during COVID19 pandemic showed that $53.8 \%$ (7/13) of patients who discontinued treatment with hydroxychloroquine did so because of drug shortage [27]. A study analysed 2311 reports from a wide range of sources, including factchecking agency websites, Facebook, Twitter, websites for television networks, and newspapers on COVID-19 in 25 languages from 87 countries between 21 January 2020 and 5 April 2020 [28]. A total of 2049 (89\%) of the reports were classified as rumours, $182(7.8 \%)$ were conspiracy theories, and $82(3.5 \%)$ were classified as stigmatization of an affected group [28].

However, it should be noted that social media platforms such as the Facebook, Twitter, YouTube, and Reddit have taken measures for dealing with misinformation such as double-check by editors, less visibility for repeated offenders, deleting of accounts and bots, as well as adding a link to official information from WHO to distribute the updates [2]. Furthermore, the impact of COVID-19 depends on the actions of individual people. A study in the USA with more than 1700 people showed that adults share false statements about COVID-19 partly because they think insufficiently about the validity of the content before sharing [29]. Therefore, both individuals, who may not have the appropriate scientific knowledge, and social media that have the power of spreading information quickly, should confront these dynamic social and scientific processes dutifully.

In the website of WHO, there are several steps to identify and avoid false information [30]. Individuals are advised to evaluate the source of information, recognize author's name, and check the date of the article. Moreover, when reading an article people should read more than just the headline and try to get a better picture of the content through diversifying their sources [30].

Apart from individuals, policy makers and the scientific community should step up to lead the fight against the growing tide of false and misleading information that threatens to worsen the severe impact of the novel coronavirus on the community. Transparency, diplomacy, and collaboration should be promoted. Negotiations between governments and technology companies could further block the distribution of false news. However, this should be implemented very carefully since the balance between preventing the propagation of false news and censorship of the press is fragile. In parallel, information campaigns should be organized to properly educate the population about the pandemic and the measurements that should be taken. In addition, the scientific community should be strengthened. This can be performed through increasing the funds for research on COVID-19, appointment of new personnel, as well as distribution of grants and scholarships.

\section{Conclusion}

During the COVID-19 pandemic, the quantity of new data is augmenting while the quality might be questionable. The main reason for this situation is the rush to quickly publish COVID-19-related articles by the authors or the journal editors and review teams. The media have an essential role in the distribution of false information; however, individual people and policy makers are also responsible. As disinformation thrives in crisis periods, well-designed studies are needed to flatten the infodemic curve regarding prevention, diagnosis, and long-term complications of COVID-19.

Funding None.

\section{Compliance with ethical standards}

Conflict of interest The authors declare they have no conflict of interest.

\section{References}

1. World Health Organization (2020) Coronavirus disease (COVID-19) dashboard. 2020. https://covid19. who.int. Accessed 16 Nov 2020

2. Marin L (2020) Three contextual dimensions of information on social media: lessons learned from the COVID-19 infodemic. Ethics Inf Technol 1-8

3. World Health Organization (2020) Munich security conference. https://www.who.int/director-general/speeches/detail/munic h-security-conference. Accessed 16 Nov 2020

4. World Health Organization (2020) Ebola virus disease-democratic Republic of the Congo. 2020. https://www.who.int/csr/ don/28-november-2019-ebola-drc/en/. Accessed 16 Nov

5. Person B, Sy F, Holton K, Govert B, Liang A. National center for inectious diseases SCOT. Fear and stigma: the epidemic within the SARS outbreak. Emerg Infect Dis. 2004;10(2):358-63.

6. Mahajan AP, Sayles JN, Patel VA, et al. Stigma in the HIV/ AIDS epidemic: a review of the literature and recommendations for the way forward. AIDS. 2008;22(Suppl 2):S67-79.

7. Kambhampati SBS, Vaishya R, Vaish A. Unprecedented surge in publications related to COVID-19 in the first three months of pandemic: a bibliometric analytic report. J Clin Orthop Trauma. 2020;11(Suppl 3):S304-6.

8. Yeo-Teh NSL, Tang BL (2020) An alarming retraction rate for scientific publications on Corsonavirus disease 2019 (COVID19). Account Res 1-7

9. Fidahic M, Nujic D, Runjic R, et al. Research methodology and characteristics of journal articles with original data, preprint articles and registered clinical trial protocols about COVID-19. BMC Med Res Methodol. 2020;20(1):161.

10. Elmore SA. Preprints: what role do these have in communicating scientific results? Toxicol Pathol. 2018;46(4):364-5.

11. Powell K. Does it take too long to publish research? Nature. 2016;530(7589):148-51. 
12. Majumder MS, Mandl KD. Early in the epidemic: impact of preprints on global discourse about COVID-19 transmissibility. Lancet Glob Health. 2020;8(5):e627-30.

13. @WHO (2020) 26 Jan 2020. https://twitter.com/WHO/statu s/1221475167869833217. Accessed 16 Nov 2020

14. Glasziou PP, Sanders S, Hoffmann T. Waste in covid-19 research. BMJ. 2020;369:m1847.

15. Martins RS, Cheema DA, Sohail MR. The pandemic of publications: are we sacrificing quality for quantity? Mayo Clin Proc. 2020;95(10):2288-90.

16. Besancon L, Peiffer-Smadja N, Segalas C, et al. (2020) Open science saves lives: lessons from the COVID-19 pandemic. https://www.biorxiv.org/content/https://doi. org/10.1101/2020.08.13.249847v1.full.pdf. Accessed 16 Nov 2020

17. Pradhan P, Pandey AK, Mishra A, et al. Uncanny similarity of unique inserts in the 2019-nCoV spike protein to HIV-1 gp120 and Gag (withdrawn). bioRxiv 2020; published online 31 Jan. doi:https://doi.org/10.1101/2020.01.30.927871

18. Strojil J, Suchankova H. Lessons for teaching from the pandemic. Br J Clin Pharmacol. 2020. https://doi.org/10.1111/bcp.14529.

19. Elavarasi A, Prasad M, Seth T, et al. Chloroquine and hydroxychloroquine for the treatment of COVID-19: a systematic review and meta-analysis. J Gen Intern Med. 2020. https://doi. org/10.1007/s11606-020-06146-w.

20. Retracted coronavirus (COVID-19) papers (2020) https://retra ctionwatch.com/retracted-coronavirus-covid-19-papers/. Accessed 16 Nov 2020

21. The Editors of the Lancet $\mathrm{G}$ (2020) Learning from a retraction. Lancet 396(10257): 1056

22. Soltani P, Patini R. Retracted COVID-19 articles: a side-effect of the hot race to publication. Scientometrics. 2020;125:1-4.

23. Trikalinos NA, Evangelou E, Ioannidis JP. Falsified papers in high-impact journals were slow to retract and indistinguishable from nonfraudulent papers. J Clin Epidemiol. 2008;61(5):464-70.
24. Vosoughi S, Roy D, Aral S. The spread of true and false news online. Science. 2018;359(6380):1146-51.

25. Busari S, Adebayo B. (2020) Nigeria records chloroquine poisoning after trump endorses it for coronavirus treatment. CNN. https ://edition.cnn.com/2020/03/23/africa/chloroquine-trump-niger ia-intl/index.html. Accessed 16 Nov 2020

26. Spencer SH (2020) False claims of nationwide lockdown for COVID-19. Factcheck. https://www.factcheck.org/2020/03/false -claims-of-nationwide-lockdown-for-covid-19/. 2020. Accessed 16 Nov 2020

27. Fragoulis GE, Evangelatos G, Arida A, et al. Treatment adherence of patients with systemic rheumatic diseases in COVID-19 pandemic. Ann Rheum Dis. 2020. https://doi.org/10.1136/annrh eumdis-2020-217935.

28. Islam MS, Sarkar T, Khan SH, et al. COVID-19-related infodemic and its impact on public health: a global social media analysis. Am J Trop Med Hyg. 2020;103(4):1621-9.

29. Pennycook G, McPhetres J, Zhang Y, Lu JG, Rand DG. Fighting COVID-19 misinformation on social media: experimental evidence for a scalable accuracy-nudge intervention. Psychol Sci. 2020;31(7):770-80.

30. World Health Organization (2020) https://www.who.int/newsroom/spotlight/let-s-flatten-the-infodemic-curve. Accessed 16 Nov 2020

Publisher's Note Springer Nature remains neutral with regard to jurisdictional claims in published maps and institutional affiliations. 